\title{
Modeling Underwater Acoustic Channels in Short-range Shallow Water Environments
}

\author{
Hovannes Kulhandjian \\ Department of Electrical Engineering \\ State University of New York at Buffalo \\ Buffalo, NY 14260 \\ hkk2@buffalo.edu
}

\author{
Tommaso Melodia \\ Department of Electrical and Computer Engineering \\ Northeastern University \\ Boston, MA 02115 \\ melodia@ece.neu.edu
}

\begin{abstract}
We analyze the statistical channel properties of short to very shortrange shallow water communication environments based on real channel measurements taken in a water-tank, a swimming pool, very shallow and shallow lakes. More specifically, we estimate the channel impulse response (CIR), the probability density function (PDF) of channel fading and fit to Rayleigh, Nakagami, Weibull, Rician and Beta distributions. We compare the 'goodness-of-fit' of these distributions based on the Kullback-Leibler (KL) divergence criteria. From our experimental results, we confirm that the shallow water acoustic channel is highly time-varying and does not necessarily follow a Rayleigh distribution. Instead, we observe that in very-shallow water lake environments the channel fading exhibits close-to Weibull or Rician distribution. On the other hand, in shallow water lake the channel fading behavior is better captured by a Beta distribution.
\end{abstract}

\section{INTRODUCTION}

Statistical modeling of radio frequency (RF) wireless communication in air has been well studied, and widely accepted channel models are available. Because of the high complexity of the underwater acoustic channel [1] it is very difficult to come up with a single statistical channel model that can be used to capture different underwater channel environments. Moreover, conducting underwater experiments and collecting data is very costly [1]. Accordingly, there are only a few joint venture underwater experiments conducted to collect underwater data, which are not readily available. In addition to that, only very limited work has been done to study the characteristics of the underwater acoustic channel in shallow water environments.

Among these, in [2], the authors study the statistical characteristics and evaluate the capacity of shallow water acoustic communication channels. Wideband single-carrier and multi-carrier probe signals are used to measure the time-varying channel response and estimate its statistical properties. From the experimental data col-

\footnotetext{
Acknowledgment: This work is based upon work supported in part by the US National Science Foundation under grants CNS1422874 and CNS-1126357.

Permission to make digital or hard copies of all or part of this work for personal or classroom use is granted without fee provided that copies are not made or distributed for profit or commercial advantage and that copies bear this notice and the full citation on the first page. To copy otherwise, to republish, to post on servers or to redistribute to lists, requires prior specific permission and/or a fee.

WUWNet'14, Nov. 12 - 14, 2014 Rome, Italy.

Copyright 2014 ACM 978-1-4503-3277-4/14/11 ...\$15.00

http://dx.doi.org/10.1145/2671490.2674560
}

lected in the Pacific Ocean, it is shown that Rician channel fading provides a good match for the experimental data.

In [3], shallow water acoustic channel measurements are presented. The authors study the temporal variability of the multipath arrival structure. In addition, they analyze the ambient noise characteristics in warm shallow water channels.

In [4], channel characteristics in a very shallow estuary are studied. The authors study the multipath intensity profile, coherence time, coherence bandwidth, the channel's scattering function, and Doppler power spectrum. Maximum likelihood estimation is used to fit the fading channel model obtained from experimental data to Rayleigh, Rice, and Nakagami-m distributions. Experimental results reveal that the channel fading may not necessarily follow a Rayleigh distribution over increasing distances.

In [5], channel measurements were performed to characterize shallow-water acoustic propagation channels in northern Europe, including Norwegian fjords, a sheltered bay and the Baltic Sea. Experiments were conducted using different frequency bands between $2 \mathrm{kHz}$ and $32 \mathrm{kHz}$. The experiment results reveal that various channels behave differently and that it is difficult to define a typical acoustic communication channel.

In [6], an experimental study is conducted to analyze the spacetime correlation and power-delay profile (PDP) properties of the underwater acoustic channel near the Pianosa Island, off the northwestern coast of Italy. Measured channel impulse responses are compared against the ones obtained from the Bellhop ray tracing tool, which are shown to closely match.

In [7], collected data in shallow water sea is analyzed under different conditions to illustrate how the ocean environments (sea surface waves and random ocean medium) affect the signal properties. Among some of the properties analyzed in this work are the amplitude and phase variations, and temporal coherence of individual paths as well as the temporal and spatial coherence of multipaths at different time scales.

In [8], statistical characteristics including the PDF and secondorder statistics of underwater acoustic channels are estimated using ocean experimental data. Kolmogorov-Smirnov test is used to measure how well the experimental PDF fits to the Gamma, Rayleigh, and compound-K distributions. From the experimental results the authors show that the underwater acoustic channel does not necessarily follow a Rayleigh distribution.

In this work, we contributed to this growing body of work by presenting and analyzing the experimental data that we have collected during our underwater testbed deployments and analyze the channel characteristics of several short-range shallow water communication environments. Specifically, we study the channel characteristics in a water-tank, a swimming pool, very shallow and shallow lakes. The channel measurements were collected during our underwater experiments at the University at Buffalo and 
Lake Erie, Buffalo. We estimate the channel impulse response (CIR), the probability density function (PDF) of channel fading and fit to a Rayleigh, Nakagami, Weibull, Rician and Beta distributions. Kullback-Leibler (KL) divergence criteria is used to test the 'goodness-of-fit' of the measured channel PDF to the theoretical PDFs.

The rest of the paper is organized as follows. In Section 2, we discuss the underwater channel characteristics. In Section 3, we present the experimental results of channel characteristics in four different environments, a water-tank, a swimming pool, very shallow and shallow lakes. Finally, in Section 4, we draw the main conclusions.

\section{CHANNEL CHARACTERISTICS}

Shallow water ${ }^{1}$ acoustic communications are characterized by the long delay spread caused by the multipath effects due to reflections from the surface and the bottom of the medium. Moreover, the dynamic channel environment caused by the motion of acoustic transducers, ocean floor, internal and surface waves results in long time variations and as a consequence leads to a high Doppler spread [1].

\subsection{Multipath}

Multipath arises from either wave reflections from the surface, bottom and other objects, or wave refraction caused by sound speed variations with depth (acoustic waves always bend towards regions where the propagation speed is lower) $[9,10]$. Multipath propagation can severely deteriorate the acoustic signal, as it generates inter-symbol interference (ISI) [11, 12]. The multipath geometry depends on the link configuration. Vertical channels typically have little time dispersion, while horizontal channels may show long multipath spreads [1]. The extent of spreading is highly dependent on depth and distance between transmitter and receiver. The channel impulse response for a time-varying multipath underwater acoustic channel can be expressed as [13, 14]

$$
h(\tau, t)=\sum_{m=1}^{M} a_{m}(t) \delta\left(\tau-\tau_{m}(t)\right),
$$

where $a_{m}(t)$ and $\tau_{m}(t)$ denote time-varying path amplitude and time-varying path delay respectively, for each resolvable multipath $m=1,2, \ldots, M$ where $M$ is the total number of multipath. In Sec. 3 we estimate the CIR and study the multipath in four different environments.

\subsection{Channel Fading Models}

In wireless RF communication Rayleigh distribution is often used to model the multipath propagation. It is well known that when the PDF of the CIR follows a zero mean white Gaussian distribution then the envelop of the channel response follows a Rayleigh distribution [8].

The probability density function of the Rayleigh distribution is expressed by [15]

$$
f(x ; \sigma)=\frac{x}{\sigma^{2}} \exp \left(-\frac{x^{2}}{2 \sigma^{2}}\right), \quad x \geq 0,
$$

where $\sigma^{2}=E(x) / 2$ and $\sigma>0$, is the scale parameter of the distribution.

In addition to Rayleigh distribution there are several other models often used to model channel fading. We will mainly focus on

\footnotetext{
${ }^{1}$ In oceanic literature shallow water environment is typically referred to a body of water with depth lower than $100 \mathrm{~m}$, while deep water is used for deeper oceans [1]. In this work very shallow water is referred to a body of water with depth less than $10 \mathrm{~m}$.
}

Nakagami, Weibull, Rician and Beta distributions. Although Beta distribution is not very common for channel modeling, we will show in Sec. 3.4.1 that it can be a better candidate to model certain classes of shallow water channels.

The probability density function of the Nakagami distribution is expressed by [15]

$$
f(x ; m, \Omega)=\frac{2 m^{m}}{\Gamma(m) \Omega^{m}} x^{2 m-1} \exp \left(-\frac{m}{\Omega} x^{2}\right),
$$

where $\Gamma(m)=\frac{x^{2 m}}{\sigma^{m}}$ and $\Omega=\frac{x^{2}}{m}$, in which $m$ is a shaping parameter and $\Omega$ controls the spread.

The probability density function of the Weibull distribution is expressed by [15]

$$
f(x ; \lambda, k)= \begin{cases}\frac{k}{\lambda}\left(\frac{x}{\lambda}\right)^{k-1} e^{-(x / \lambda)^{k}} & x \geq 0, \\ 0 & x<0,\end{cases}
$$

where $k>0$ is the shape parameter and $\lambda>0$ is the scale parameter of the distribution.

The probability density function of the Rician distribution is expressed by [15]

$$
f(x \mid \nu, \sigma)=\frac{x}{\sigma^{2}} \exp \left(\frac{-\left(x^{2}+\nu^{2}\right)}{2 \sigma^{2}}\right) I_{0}\left(\frac{x \nu}{\sigma^{2}}\right),
$$

where $I_{0}(z)$ is the modified Bessel function of the first kind with order zero.

The probability density function of the Beta distribution is expressed by [15]

$$
\begin{aligned}
f(x ; \alpha, \beta) & =\frac{\Gamma(\alpha+\beta)}{\Gamma(\alpha) \Gamma(\beta)} x^{\alpha-1}(1-x)^{\beta-1} \\
& =\frac{1}{B(\alpha, \beta)} x^{\alpha-1}(1-x)^{\beta-1}
\end{aligned}
$$

where $\Gamma(z)$ is the gamma function and $B(\alpha, \beta)=\frac{\Gamma(\alpha) \Gamma(\beta)}{\Gamma(\alpha+\beta)}$ is a normalization constant to ensure that the total probability integrates to 1 .

In Sec. 3 we study the experimental PDF of channel fading in different environments and fit to Rayleigh, Nakagami, Weibull, Rician and Beta distributions. To test how well the existing statistical models fit the experimental PDF we make use of Kullback-Leibler divergence criteria, which is asymptotically unbiased [16].

\subsection{Kullback-Leibler Divergence}

Kullback-Leibler (KL) divergence is a non-symmetric measure of the difference between two probability distributions. It measures the lack of fit between a model and data relative to a perfect fit [16]. KL divergence or relative entropy between two probability mass vectors $\boldsymbol{p}$ and $\boldsymbol{q}$ can be expressed as [17]

$$
D(\boldsymbol{p} \| \boldsymbol{q})=\sum_{x \in X} p(x) \log \left(\frac{p(x)}{q(x)}\right) .
$$

$D(\boldsymbol{p} \| \boldsymbol{q})$ measures the "distance" between the probability mass function $p(x)$ and $q(x)$ for a random variable $X$. Typically, $p(x)$ represents the "true" data distribution, while $q(x)$ represents an approximation of $p(x)$. The smaller the KL divergence is the closer the two distributions are (i.e., $\mathrm{KL}=0$ means $p(x)=q(x)$ ).

In Sec. 3 we use KL divergence to test how well the experimental PDF fits to the statistical PDF models discussed in Sec. 2.2.

\section{EXPERIMENTAL RESULTS}

We consider four different underwater channel environments namely a water tank, a swimming pool, a very shallow water lake and a deeper lake. The experimental results are presented in the following. 


\subsection{Underwater Lab Experiments}

The first set of underwater experiments were conducted in a water tank of dimensions $2.5 \mathrm{~m} \times 0.7 \mathrm{~m} \times 0.6 \mathrm{~m}$ in the Underwater Acoustic laboratory at the State University of New York at Buffalo, using three Telesonar SM-75 SMART modems by Teledyne Benthos [18], as shown in Fig. 1. The omnidirectional transducer operates in the $9-14 \mathrm{kHz}$ (LF) low frequency band. The waveformplay feature of the modem enables transmissions of baseband complex data with a bandwidth of $5,120 \mathrm{~Hz}$ sampled at $10,240 \mathrm{~Hz}$. The data packets were generated using Matlab, converted into a stereo WAV file in 16 bit format, and uploaded on the modems through the RS-232 interface. Each data packet contains a preamble, a chirp signals of duration $100 \mathrm{~ms}$ sweeping the bandwidth from $10 \mathrm{~Hz}$ to $5.12 \mathrm{kHz}$. The chirp signal is appended to each packet and is used for channel probing, symbol synchronization for chip-matched filtering, and multipath delay spread estimation [19]. The chirp signals are in baseband and are modulated by multiplying by the carrier $f_{c}$ before transmission. Data bits are modulated using direct-sequence code-division multiple-access (DS-CDMA), which is used for estimating the channel fading. The DS-CDMA chip waveforms are selected from the columns of a Sylvester-Hadamard matrix of order $L=32$ with a chip rate $R_{c}=2,048$ chips $/ \mathrm{sec}$. Pulse shaping was done using square-root raised-cosine with rolloff factor $\beta=0.5$. A total of $1.25 \mathrm{kByte}$ of data per packet was transmitted using the lowest transmit power level (i.e., $1.78 \mathrm{~W}$ ) and each experiment was repeated 20 times with 5 min. intermission window. The packets were transmitted by the modem located in the middle, and were received by the two modems on either side separated by about one meter from the transmitter. Because of the symmetric testbed setup, the two channels showed similar characteristics. Thus, we only present the channel characteristics from the transmitter to the left side receiver. Each modem is equipped with a data recorder that has a storage capacity of 64 GBytes. The raw data were recorded and analyzed offline. The ensemble average statistics are presented in the plots.

\subsubsection{Water Tank Channel Characteristics}

Using the chirp signal we study the CIR in the water tank environment, which is plotted in Fig. 2 by matched filtering the transmitted chirp signal with the received preamble. We observe that the channel is prone to strong multipath effect with a delay spread of $3 \mathrm{~ms}$. What is interesting to note is that due to the confined walls of the water tank several multipath components, located close to each other, carry comparable amount of signal power, which can generate ISI as well as inter-chip-interference (ICI), and thus may require complex receiver design.

Next, we study the PDF of the DS-CDMA complex received signal magnitude, which is plotted in Fig. 3, using 100 bin values. Since the transmitter and receiver are in very close proximity of each other, the received signal power is relatively high compared to the noise floor (i.e., high signal-to-noise (SNR)). Because of the lack of noise component, the received signal amplitude does not experience randomness, accordingly, the experimental PDF does not necessarily follow any of the PDF models discussed in Sec. 2.2.

Using the KL distance criteria, shown in Table 1, we can see that the closest approximation is Weibull or Rician distribution, which are shown in bold.

\subsection{Swimming Pool Experiments}

The next set of experiments were conducted in the diving pool of the Alumni Arena at the University at Buffalo as shown in Fig. 4. The dimensions of the diving pool are $22 \mathrm{~m} \times 16 \mathrm{~m} \times 4.9 \mathrm{~m}$ (length, width and depth). On one side of the pool an acoustic transducer, connected to the surface station universal deck box (UDB-9000)
Table 1: KL divergence for five different distributions.

\begin{tabular}{|l|c|c|c|c|c|}
\hline Distribution: & Rayleigh & Nakagami & Weibull & Rician & Beta \\
\hline Water tank & 1.7 & 1.5 & $\mathbf{0 . 9 2}$ & $\mathbf{0 . 9 4}$ & 1.3 \\
\hline Swimming pool & 0.86 & 0.68 & $\mathbf{0 . 4 7}$ & $\mathbf{0 . 5 2}$ & 0.64 \\
\hline Lake LaSalle & 0.36 & 0.21 & $\mathbf{0 . 0 7}$ & $\mathbf{0 . 0 4}$ & 0.62 \\
\hline Lake Erie & 0.24 & 0.21 & 0.20 & 0.24 & $\mathbf{0 . 0 2}$ \\
\hline
\end{tabular}

and controlled by a laptop through the RS-232 interface, was submerged to a depth of $2 \mathrm{~m}$ from the water surface. On the far end of the pool an SM-75 modem with a data recorder was immersed again to a depth of $2 \mathrm{~m}$ from the surface of the pool. The distance between the transmitter and the receiver was about $20 \mathrm{~m}$. The same data packets discussed in Sec. 3.1 were transmitted by the UDB9000 and each experiment was repeated 20 times with 5 min. intermission window. The experiments were conducted at $3 \mathrm{~W}$ transmit power level. The raw data were recorded and analyzed offline. The ensemble average statistics are presented in the plots.

\subsubsection{Swimming Pool Channel Characteristics}

The channel impulse response is shown in Fig. 5. We may observe that the channel is prone to high multipath effects with a delay spread of $7.5 \mathrm{~ms}$. A large number of multipath components with fairly high magnitude are observed near the line-of-sight (LOS) component, because of the reflections from the wall of the swimming pool, which again will generate high ISI and ICI.

The PDF of the complex received signal magnitude is plotted in Fig. 6. Interestingly, the Rayleigh distribution is not the best candidate to represent the channel fading. Instead, we can see that the closest approximation are Weibull and Rician distributions, which is also confirmed using the KL distance criteria, shown in Table 1.

\subsection{Lake LaSalle Experiments}

The next set of experiments were conducted in Lake LaSalle at the University at Buffalo using two Telesonar SM-75 modems, which were deployed $2 \mathrm{~m}$ below the surface separated by about $110 \mathrm{~m}$ from each other. The average depth of the lake was about $4.5 \mathrm{~m}$. The actual deployment of the modems is shown in Fig. 7, with two orange buoys floating on the surface of the lake, each attached to the Telesonar SM-75 modem along with an anchor. Alice transmitted the same data packets discussed in Sec. 3.1 to Bob at 7.1 W transmit power level and each experiment was repeated 20 times with $5 \mathrm{~min}$. intermission window. A laptop, on an inflatable boat, was used to coordinate the transmissions of the packets through a serial port interface. The raw data were recorded and analyzed offline. The ensemble average statistics are presented in the plots.

\subsubsection{Lake LaSalle Channel Characteristics}

Figure 8 shows the normalized CIR amplitude obtained by chirpmatched filtering the received acoustic signal at Bob. We observe that the channel is prone to high multipath effects with a multipath delay spread of about $14 \mathrm{~ms}$. In addition, we observe that some of the multipath components are within the chip period $(0.5 \mathrm{~ms})$, which may cause ICI as well as ISI. Compared to the water tank and the swimming pool channels the delay spread is much longer, due to the very shallow water and longer communication range.

The PDF of the complex received signal magnitude is plotted in Fig. 9, in which again we can see that the Rayleigh distribution may not be the best candidate to represent the channel fading. Instead, we observe that the closest approximations are Weibull and Rician distributions, which is also confirmed using the KL distance criteria, shown in Table 1. 


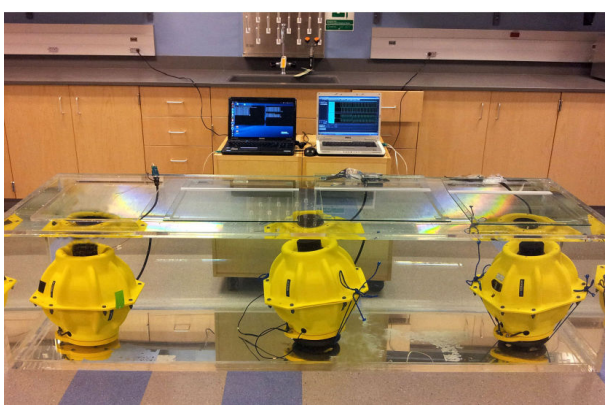

Figure 1: Channel measurements in a water tank at the University at Buffalo.

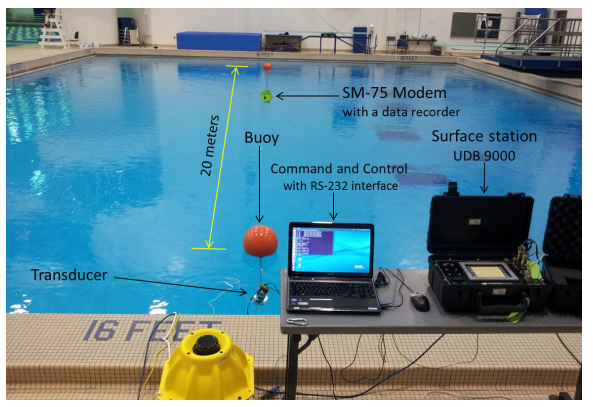

Figure 4: Channel measurements in the diving pool at the University at Buffalo.

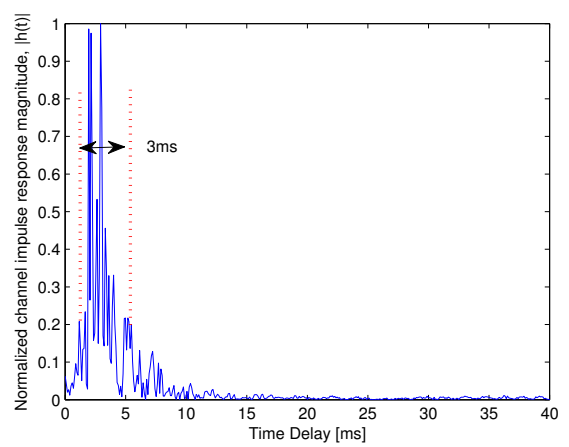

Figure 2: Channel impulse response in the water tank.

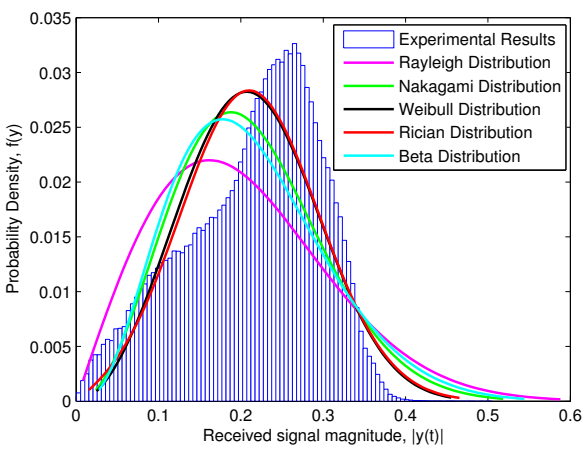

Figure 3: Received signal PDF in the water tank.

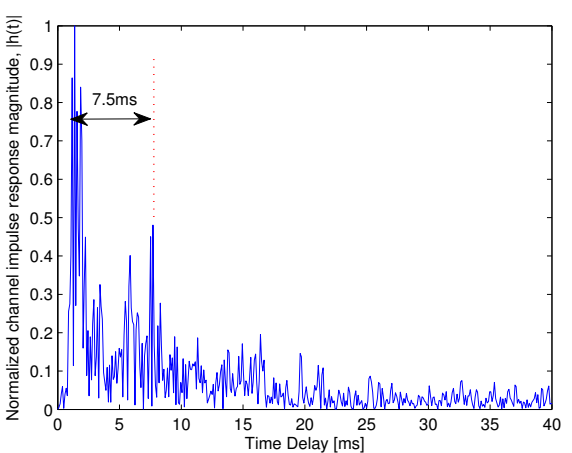

Figure 5: Channel impulse response in the swimming pool.

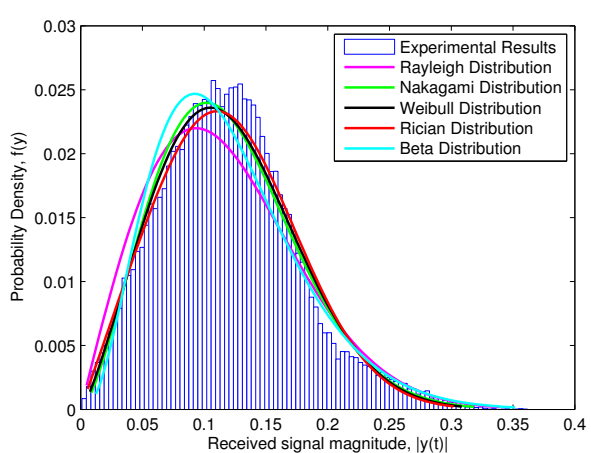

Figure 6: Received signal PDF in the swimming pool.

\subsection{Lake Erie Experiments}

The last set of experiments were conducted in Lake Erie a few miles south of downtown Buffalo, New York. The actual deployment is shown in Fig. 10, with two Telesonar SM-75 modems deployed $2 \mathrm{~m}$ below the surface separated by about $200 \mathrm{~m}$ from each other. The average depth of the lake at the experiment location was about $15 \mathrm{~m}$. A laptop on a boat was used to coordinate the transmissions of the packets through a serial port interface. Alice transmitted the same data packets discussed in Sec. 3.1 to Bob at $14.2 \mathrm{~W}$ transmit power level and each experiment was repeated 20 times with 5 min. intermission window. The ensemble average statistics are presented in the plots.

\subsubsection{Lake Erie Channel Characteristics}

Figure 11 shows the normalized CIR amplitude obtained by chirp-matched filtering the received acoustic signal at Bob. The multipath channel at Lake Erie is less severe compared to the other channels we have investigated in this work. Although the channel experiences slightly longer multipath delay spread of about $15 \mathrm{~ms}$, there are only very few multipath components, which can readily be resolved. Therefore, the channel will not generate ICI, but may result in ISI depending on the signal symbol duration used.

The PDF of the complex received signal magnitude is plotted in Fig. 12, in which again we can see that the Rayleigh distribution, which in this case is identical to the Rician distribution, may not be the best candidate to represent the channel fading. Interestingly, we can observe that the PDF for this channel is better captured by a Beta distribution, which is also confirmed using the KL distance criteria, shown in Table 1.

\section{CONCLUSIONS}

We presented and analyzed the statistical channel characteristics of short to very short-range shallow water communication environments, including, a water-tank, a swimming pool, very shallow and shallow lakes. Our experimental results confirm that the shallow water acoustic channel is highly time-varying and does not necessarily follow a Rayleigh distribution. Instead, we observe that in very-shallow water lake environments the channel fading exhibits close-to Weibull or Rician distribution. On the other hand, in shallow water lake the channel fading behavior is better captured by a Beta distribution.

\section{REFERENCES}

[1] T. Melodia, H. Kulhandjian, L. Kuo, and E. Demirors, "Advances in underwater acoustic networking," in Mobile Ad Hoc Networking: Cutting Edge Directions, S. Basagni, M. Conti, S. Giordano, and I. Stojmenovic, Eds. Inc., Hoboken, NJ: John Wiley and Sons, 2013, pp. 804-852.

[2] A. Radosevic, J. G. Proakis, and M. Stojanovic, "Statistical characterization and capacity of shallow water acoustic channels," in Proc. of MTS/IEEE OCEANS, Biloxi, MS, USA, October 2009.

[3] M. Chitre, J. Potter, and O. S. Heng, "Underwater acoustic channel characterisation for medium-range shallow water communications," in Proc. of MTS/IEEE OCEANS, vol. 1, Kobe, Japan, Nov. 2004, pp. 40-45.

[4] B. Borowski, "Characterization of a very shallow water acoustic communication channel," in Proc. of MTS/IEEE OCEANS, Biloxi, MS, USA, October 2009, pp. 1-10. 


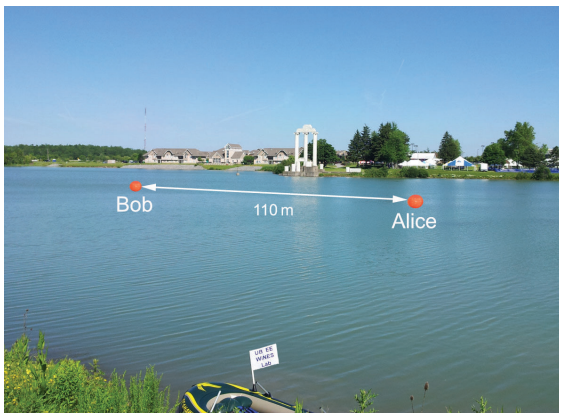

Figure 7: Channel measurements in Lake LaSalle at the University at Buffalo.

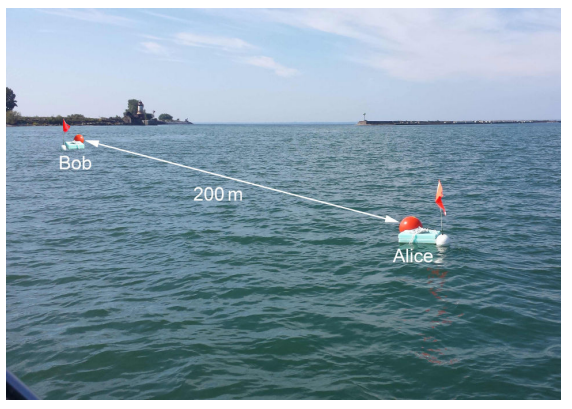

Figure 10: Channel measurements in Lake Erie, Buffalo, NY.

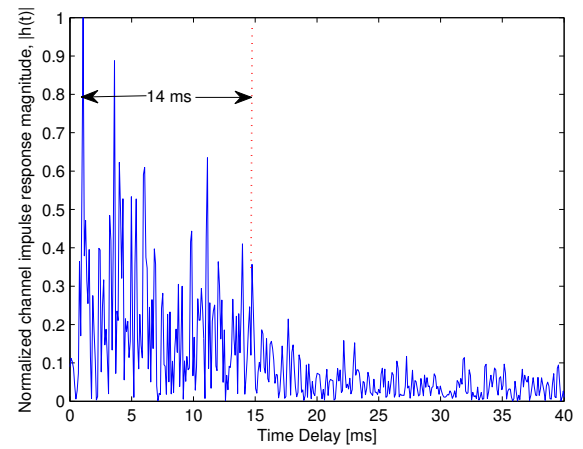

Figure 8: Channel impulse response in Lake LaSalle.

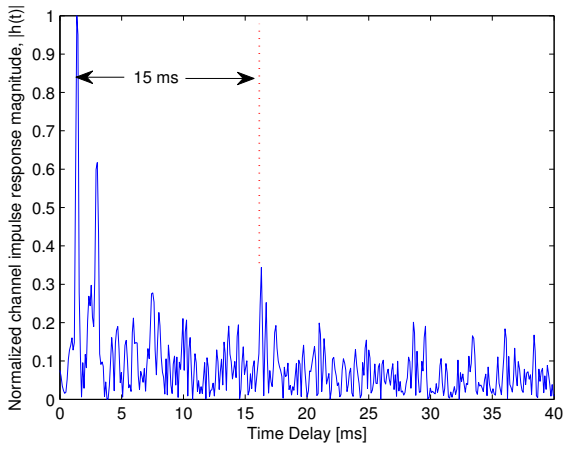

Figure 11: Channel impulse response in Lake Erie.

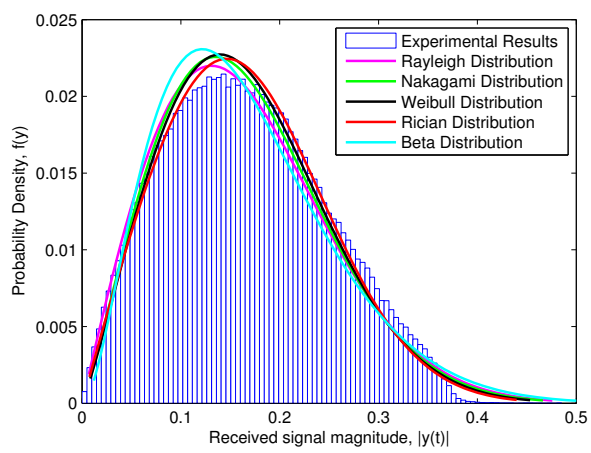

Figure 9: Received signal PDF in Lake LaSalle.

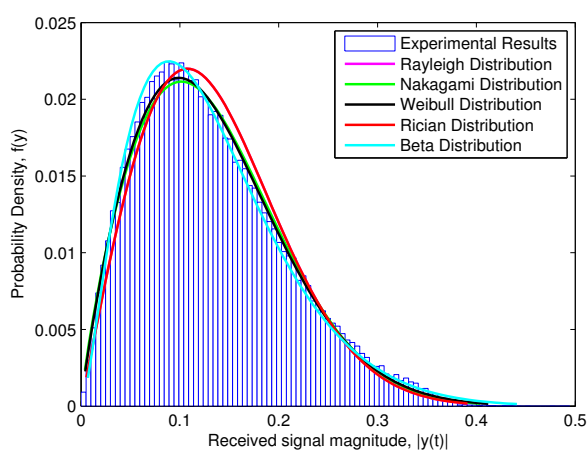

Figure 12: Received signal PDF in Lake Erie.
[5] P. A. van Walree, "Propagation and Scattering Effects in Underwater Acoustic Communication Channels," IEEE Journal of Oceanic Engineering, vol. 38, no. 4, pp. 614-631, May 2013.

[6] B. Tomasi, G. Zappa, K. McCoy, and P. Casari, "Experimental study of the space-time properties of acoustic channels for underwater communications," in Proc. of MTS/IEEE OCEANS, Sydney, Australia, May 2010, pp. 1-9.

[7] T. C. Yang, "Properties of underwater acoustic communication channels in shallow water," The Journal of the Acoustical Society of America, vol. 131, no. 1, pp. 129-145, January 2012.

[8] J. Zhang, J. Cross, and Y. R. Zheng, "Statistical channel modeling of wireless shallow water acoustic communications from experiment data," in IEEE Conf. on Military Communications (MILCOM), San Jose, CA, USA, Oct. 2010, pp. 2412-2416.

[9] R. J. Urick, Principles of Underwater Sound. McGraw-Hill, 1983.

[10] M. Stojanovic, "Underwater acoustic communications: Design considerations on the physical layer," in Proc. IEEE / IFIP Fifth Annual Conference on Wireless On demand Network Systems and Services (WONS 2008), Garmisch-Partenkirchen, Germany, January 2008, pp. 1-10.

[11] D. B. Kilfoyle and A. B. Baggeroer, "The State of the Art in Underwater Acoustic Telemetry," IEEE Journal of Oceanic Engineering, vol. 25, pp. 4-27, Jan. 2000.

[12] H. Kulhandjian, T. Melodia, and D. Koutsonikolas, "CDMA-based Analog Network Coding through
Interference Cancellation for Underwater Acoustic Sensor Networks," in Proc. of ACM Intl. Conf. on UnderWater Networks and Systems (WUWNet), Los Angeles, CA, USA, November 2012.

[13] B. Li, S. Zhou, M. Stojanovic, L. Freitag, and P. Willett, "Multicarrier communication over underwater acoustic channels with nonuniform Doppler shifts," IEEE Journal of Oceanic Eng., vol. 33, no. 2, pp. 198-209, April 2008.

[14] H. Kulhandjian, L. Kuo, T. Melodia, D. A. Pados, and D. Green, "Towards Experimental Evaluation of Software-Defined Underwater Networked Systems," in Proc. of IEEE UComms, Sestri Levante, Italy, September 2012.

[15] A. Papoulis and S. U. Pillai, Probability, random variables, and stochastic processes. Tata McGraw-Hill Education, 2002.

[16] S. Eguchi and J. Copas, "Interpreting Kullback-Leibler divergence with the Neyman-Pearson lemma," Journal of Multivariate Analysis, vol. 97, no. 9, pp. 2034-2040, 2006.

[17] S. Kullback and R. A. Leibler, "On information and sufficiency," The Annals of Mathematical Statistics, vol. 22, no. 1, pp. 79-86, March 1951.

[18] Teledyne-Benthos, Acoustic Modems. [Online]. Available: http://www.benthos.com.

[19] H. Kulhandjian, T. Melodia, and D. Koutsonikolas, "Securing Underwater Acoustic Communications through Analog Network Coding," in Proc. of IEEE Intl. Conf. on Sensing, Comm., and Networking (SECON), Singapore, June 2014. 